\title{
Changing Morning Report: An Educational Intervention to Address Curricular Needs
}

\author{
Vijay John Daniels and Cheryl Eleanor Goldstein \\ Division of General Internal Medicine, University of Alberta, 5-112 Clinical Sciences Building, University of Alberta Hospital, \\ 11350-83 Avenue NW, Edmonton, AB, Canada T6G 2G3
}

Correspondence should be addressed to Vijay John Daniels; vdaniels@ualberta.ca

Received 29 August 2013; Revised 17 January 2014; Accepted 24 January 2014; Published 5 March 2014

Academic Editor: Geoffrey Lighthall

Copyright (C) 2014 V. J. Daniels and C. E. Goldstein. This is an open access article distributed under the Creative Commons Attribution License, which permits unrestricted use, distribution, and reproduction in any medium, provided the original work is properly cited.

\begin{abstract}
Morning report is a case-based teaching session common to many residency programs with varying purposes and focuses. At our institution, physicians and residents felt our Internal Medicine morning report had lost its educational focus. The purpose of this project was to improve morning report using a well-known curriculum development framework for medical education. We conducted a focus group of residents to develop and implement changes to morning report. Themes from our focus group led us to split morning report with the first 30 minutes for postgraduate year 3 (PGY-3) residents to give handover, to receive feedback on diagnosis and management, and to either discuss an interesting case or receive teaching aimed at their final certification examination. The second 30 minutes involved PGY-3 residents leading PGY-1 residents in case-based discussions with an attending physician providing feedback on the content and process of teaching. We measured success based on a follow-up survey and comments from resident evaluations before and after the change. Overall, the changes were well received by both faculty and residents; however comments revealed that the success of morning report is preceptor dependent. In summary, we have successfully implemented a split morning report model to enhance resident education with positive feedback.
\end{abstract}

\section{Introduction}

Morning Report is a case-based teaching session that occurs in residency programs across North America [1,2], Europe [3, $4]$, and Asia $[5,6]$. Though there is evidence that discussing patients at morning report can improve patient outcomes such as length of stay [7], the primary purpose of morning report across centers has been education $[2,8]$. In the last ten years since the detailed review by Amin and colleagues, much research has focused on various methods and innovations to enhance the educational value of morning report [917], but few articles describe explicit links to residency curriculum objectives [18-20]. At the University of Alberta Hospital, a tertiary care facility, evaluations of the Internal Medicine rotation, and discussions in our General Internal Medicine divisional meetings indicated that both residents and attending physicians felt that our one-hour morning report was not aligned with our curriculum and had lost its educational focus, with the majority of time spent on the handover of patients admitted overnight. The purpose of this project was to restore the educational focus of morning report and to align it with the goals of our curriculum.

\section{Materials and Methods}

We used the curriculum development framework for medical education by Kern and colleagues [21] to analyze our situation and guide an intervention. The six steps of the framework are (1) problem identification and general needs assessment, (2) targeted needs assessment, (3) goals and objectives, (4) educational strategies, (5) implementation, and (6) evaluation and feedback. Our first step was to survey the literature for an ideal approach to morning report. Next, we discussed the literature review as part of a focus group with our primary audience, postgraduate year 3 (PGY-3) Internal Medicine residents. This group was chosen because they are the primary participants in morning report where they hand over the newly admitted patients they saw overnight. The focus group questions were as follows. (1) "What do you like 
about morning report?" (2) "What do you dislike?” (3) "What should be the goals of morning report?" (4) "What should we change to achieve these goals?" (5) "Here are some ideas from the literature; do any of these work?"

We performed a thematic analysis and this was used to propose changes to morning report, which were presented to another group of PGY-3 residents along with the themes for verification. We then presented the changes to our faculty for discussion. Once approved, the changes were implemented to morning report. We collected residents' comments pertaining to morning report from a year's worth of rotation evaluations before and after the changes. We also surveyed our faculty and residents who participated in both the old and new morning report format after a year to assess the new model. The survey involved respondents rating their agreement with the statement "I think the following are important additions to morning report" and then being presented with the various changes that were implemented. SPSS [22] was used for Wilcoxon signed-ranks tests to compare respondents' ratings to the neutral score to assess agreement with these statements and for MannWhitney $U$ tests to assess differences between responses from residents and attending physicians. The respondents were also asked to select which change still needs the most ongoing improvement and Fisher's exact test was used to compare differences between residents and attending physicians.

\section{Results}

3.1. Step 1: Problem Identification and General Needs Assessment. We performed a review of the literature surrounding morning report to look for a general current state and if possible an ideal state for morning report. As previously mentioned, there are a variety of different purposes and formats [9-20] but none with one clearly superior strategy, so we turned to the available literature reviews. The review by Amin and colleagues [2] discussed the current state of morning report; historically, it was created to ensure the health and safety of patients but has now evolved into primarily an educational venue with case-based presentations and discussions being the most often used format. Both this review and a more recent one by McNeill and colleagues [8] concluded that there was insufficient evidence to make firm recommendations for an ideal structure/configuration for morning report but did comment that each program should tailor morning report to fit their needs.

3.2. Step 2: Targeted Needs Assessment. Seven out of 21 PGY-3 Internal Medicine residents participated in the focus group regarding their likes and dislikes of morning report and to discuss the review of the literature to elicit desired changes to morning report. Content analysis revealed the following themes: (1) too much focus on handover with residents sharing the entire case story, not just the summary;

(2) minimal and ineffective feedback to the PGY-3 resident presenting handover in terms of diagnosis and management; (3) unclear expectations and poor learning environment with
PGY-3 residents feeling embarrassed in front of PGY-1 residents; (4) minimal teaching directed at the PGY-3 residents' level, in particular residents requesting more physical exam teaching and more experience with the oral examination format used in the Royal College of Physicians and Surgeons of Canada certification examination in Internal Medicine; (5) in terms of an educational strategy, the residents preferring the traditional case-based approach over other published methods.

3.3. Step 3: Goals and Objectives. We developed the following goals for morning report: (1) to teach efficient handover strategies and case presentation skills; (2) to allow discussion of interesting cases; (3) to orient PGY-3 residents to the oral examination format used in the Canadian certification examination in Internal Medicine; (4) to enhance physical examination skills; (5) to allow PGY-3 residents protected time to teach PGY-1 residents and receive feedback regarding their teaching. Because our morning report is limited to one hour, we acknowledged to faculty and learners that not all of these goals would be met every day, but over a four-week period, we would touch upon all of them.

Based on these goals, the following educational objectives were implemented for morning report. (1) Residents will be able to present a concise case summary highlighting the key features that support the suspected diagnosis and then lay out the management plan pertaining to the most critical issues. (2) Residents will engage in reflective practice by reviewing difficult cases seen overnight and will use other PGY-3 residents and attending physicians as consultants in the process. (3) Residents will develop an approach to the structured oral examination. (4) Residents will enhance their skills in physical examination. (5) Residents will demonstrate skills in teaching by leading case-based discussions after which they will receive formative feedback from attending physicians on content and process of teaching.

3.4. Step 4: Educational Strategies. The sixty minutes allocated to morning report were divided into two thirty-minute sessions; the first session was for the PGY-3 residents on their General Internal Medicine senior rotation and began with handover (targeting 10 minutes for on average 6-8 patients). In this section, we focused feedback on how the case summary was presented (e.g., missing elements or too much information) or on how each case was managed overnight. Then the residents decided if they had an interesting case to discuss. If they did, the educational focus was on clinical reasoning (assessing factors that supported the diagnosis or discussing common cognitive errors that may have led to a misdiagnosis) and/or clinical decision-making (discussion of ideal management in a given case). If the PGY-3 residents did not have a case in mind, the attending physician conducted a practice oral or physical examination scenario. The attending physician gave feedback on the PGY-3 resident's approach to the oral examination or physical examination.

During the second thirty minutes, the PGY-1 residents on their Internal Medicine ward rotation joined the PGY-3 residents and attending physician for a case-based discussion 
TABLE 1: Educational strategies in morning report.

\begin{tabular}{|c|c|c|c|}
\hline Objective & Morning report stage & Time & Focus of feedback \\
\hline (1) Handover & $\begin{array}{l}\text { Handover of patients admitted } \\
\text { overnight }\end{array}$ & 10 minutes & $\begin{array}{l}\text { (i) Communication } \\
\text { skills/handover } \\
\text { (ii) Diagnosis and management } \\
\text { of each case }\end{array}$ \\
\hline (2) Reflective practice & Discussion of interesting case & 20 minutes & $\begin{array}{l}\text { Clinical reasoning and clinical } \\
\text { decision-making }\end{array}$ \\
\hline (3) Oral examination & Practice oral examination & zo intmutes & Approach to oral examination \\
\hline (4) Physical exam & Physical examination teaching & & $\begin{array}{l}\text { Approach to physical } \\
\text { examination }\end{array}$ \\
\hline (5) Teaching & $\begin{array}{l}\text { PGY-3 residents teaching PGY-1 } \\
\text { residents using case-based } \\
\text { discussion }\end{array}$ & 30 minutes & $\begin{array}{l}\text { (i) Content of teaching } \\
\text { (ii) Process of teaching (e.g., } \\
\text { interactivity) }\end{array}$ \\
\hline
\end{tabular}

led by a PGY-3 resident. At the end of this session, the attending physician gave the PGY-3 residents feedback on their teaching, both in terms of content taught and in terms of process of teaching. See Table 1 for an overview.

3.5. Step 5: Implementation. The proposed changes were brought before our Internal Medicine Residency Program Committee who voted in favor of these changes. Then these were brought before the Division of General Internal Medicine, first for discussion and approval of the proposed changes and second for faculty development to ensure that faculty understood their role in morning report. The changes were implemented in the middle of the academic year with one of the authors present most days for the first few months to ensure ongoing adherence to and understanding of the new format.

3.6. Step 6: Evaluation and Feedback. One year after the changes to morning report, the 12 PGY-3 residents and 12 attending physicians who experienced morning report before and after the changes were invited to complete a survey. Ten residents and nine attending physicians responded to the survey asking about their opinion of the following changes to morning report: (1) feedback on diagnosis and management; (2) feedback on teaching ability; (3) physical exam teaching; (4) practice oral cases; (5) PGY-3 resident-specific teaching without PGY-1 residents present; (6) conciseness of handover. When asked to rate their agreement on a scale of 1 to $5(1=$ strongly disagree, $2=$ disagree, $3=$ neutral, $4=$ agree, and $5=$ strongly agree) with the statement "I think the following are important additions to morning report," respondents agreed that all the above changes were important $(P<0.05$ Wilcoxon signed-ranks test compared to the neutral score of 3 on the Likert scale). There were no differences between residents' and attending physicians' responses (Mann-Whitney $U$ test, $P$ range from 0.18 to 0.78 ). See Figure 1. When asked "what do you feel needs the most improvement?" $40 \%$ of residents chose "conciseness of handover" and $40 \%$ chose "feedback on diagnosis and management" compared with $67 \%$ and $11 \%$ of attending physicians, respectively. These differences were not statistically significant based on Fisher's exact test. See Figure 2.

When we reviewed the comments section for the rotation evaluations for the year prior to the changes, we found 12 comments referencing morning report, 10 of which were negative and all of which were related to the themes identified in our focus group. For the year after the changes, there were 14 comments about morning report with 9 being positive and 5 being negative. The positive comments were mainly about residents enjoying the new format and the opportunity to practice oral examination skills. The negative comments focused on how the success of morning report was highly dependent on which attending physicians were present.

\section{Discussion}

We successfully implemented a split morning report, a format we have not seen in the literature. The changes are favorable, but the success appears to be preceptor dependent. We are continuing to work on faculty development to keep morning report a consistent educational experience and have incorporated tips for the residents on how to present a concise handover into our rotation orientation.

This project is not without limitations. It was done at one hospital site in one Internal Medicine program with a small sample size. Another limitation is the postintervention design as well as the use of subjective self-report data. So far we have only evaluated resident and attending physician satisfaction with the morning report changes. It would be informative to assess whether we are meeting the remaining objectives as outlined above, with respect to improving the PGY-3 residents' case summarization skills, approach to the oral examination, and/or teaching abilities. Future research may include audio or video recordings of morning report during the first and last week of several rotations, which would allow us to evaluate if we are meeting these objectives. Future work could also involve a quasi-experimental design comparing multiple programs (some as intervention sites and some as controls), which would also increase sample size. 


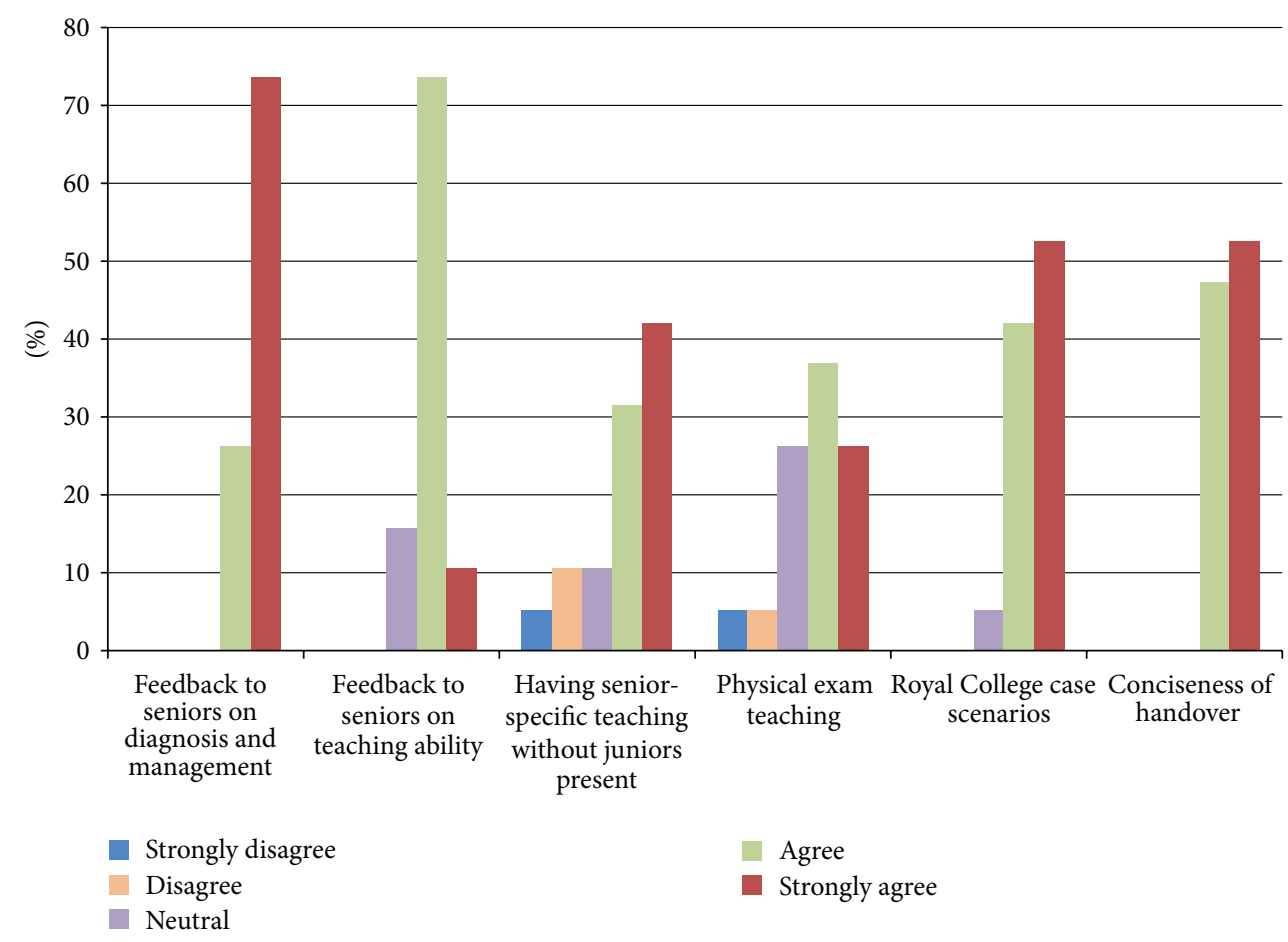

Figure 1: Important additions to morning report. Mann-Whitney $U$ tests did not reveal differences ( $P$ range is from 0.18 to 0.78 ) between residents and attending physicians and so results reported are combined residents and attending physician responses. $P<0.05$ for differences between all six items and the neutral score using Wilcoxon signed-ranks test.

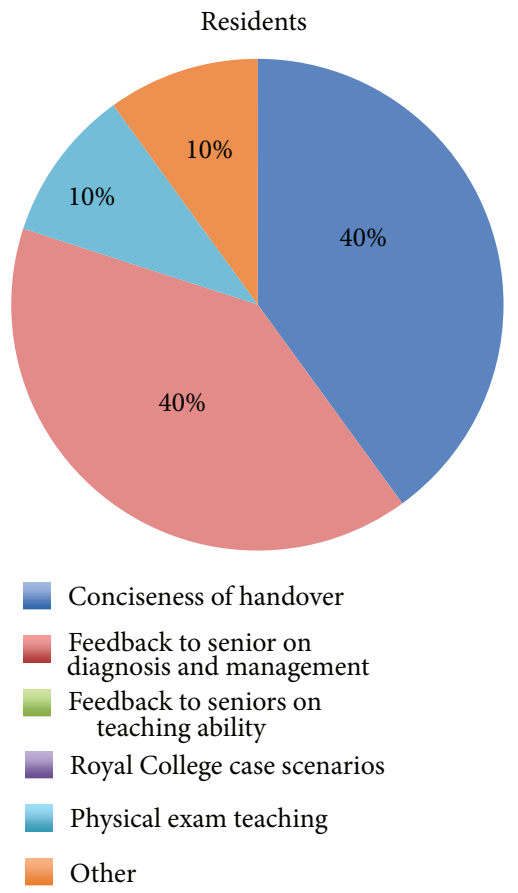

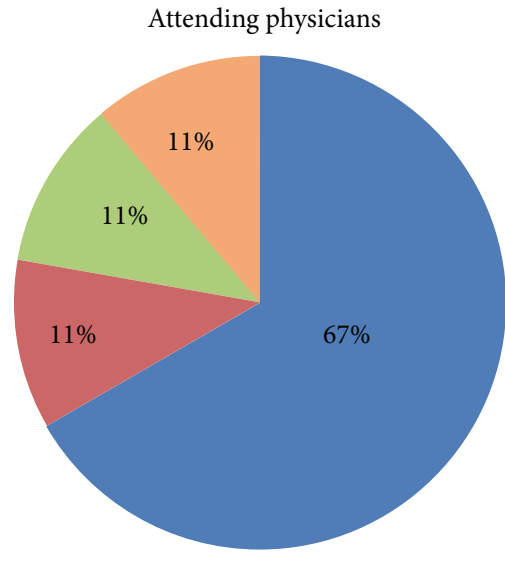

Conciseness of handover

Feedback to senior on diagnosis and management

Feedback to seniors on teaching ability

Royal College case scenarios

Physical exam teaching

Other

FIGURE 2: Still needs the most improvement. No difference between resident and attending physician responses with Fisher's exact test. 


\section{Conclusion}

Morning report is a case-based teaching session used across North America and around the world with many variations. We have successfully implemented a split morning report with a formative focus on our PGY-3 residents with positive responses from both residents and faculty. However, faculty development is an ongoing issue to maintain a consistently high quality morning report.

\section{Ethical Approval}

Ethical approval for this project was sought from the Research Ethics Board at the University of Alberta; however, this project was deemed exempt due to its program evaluation focus.

\section{Conflict of Interests}

The authors declare that no conflict of interests, financial or other, exists.

\section{Authors' Contribution}

Vijay Daniels conceived the original idea for the project and worked on the initial design with the input of Cheryl Goldstein. Vijay Daniels conducted the focus groups and created and administered the surveys. Vijay Daniels drafted the first version and subsequent revisions of the paper; Cheryl Goldstein reviewed and edited the various versions of the paper. Both authors approved the final draft.

\section{Acknowledgment}

The authors would like to thank Dr. Liam Rourke, Director of Medical Education Research in the Department of Medicine at the University of Alberta, for his input on the paper.

\section{References}

[1] T. A. Parrino and A. G. Villanueva, "The principles and practice of morning report," Journal of the American Medical Association, vol. 256, no. 6, pp. 730-733, 1986.

[2] Z. Amin, J. Guajardo, W. Wisniewski, G. Bordage, A. Tekian, and L. G. Niederman, "Morning report: focus and methods over the past three decades," Academic Medicine, vol. 75, no. 10, supplement, pp. S1-S5, 2000.

[3] J. P. Das, M. S. Draman, L. Cormican, and J. O’Neill, “Morning report: a recommendation to improve medical handover," Irish Medical Journal, vol. 105, no. 2, article 59, 2012.

[4] R. O. Gans, D. T. Sleijfer, T. S. van der Werf, and J. G. Zijlstra, "Role of the morning report in the training of internists," Nederlands Tijdschrift voor Geneeskunde, vol. 144, no. 36, pp. 1753-1754, 2000.

[5] M. Rahnavardi, B. Bikdeli, H. Vahedi et al., "Morning report: a survey of Iranian senior faculty attitudes," Internal and Emergency Medicine, vol. 3, no. 1, pp. 17-24, 2008.

[6] K. H. M. Quadri, T. Jaffery, A. Y. Alam, and F. Rahim, "Preliminary experience with a new medicine morning report format incorporating multimedia and up-to-date," Journal of the Pakistan Medical Association, vol. 57, no. 6, pp. 320-321, 2007.

[7] E. Boushehri, M. E. Khamseh, A. Farshchi, R. Aghili, M. Malek, and A. E. Valojerdi, "Effects of morning report case presentation on length of stay and hospitalisation costs," Medical Education, vol. 47, no. 7, pp. 711-716, 2013.

[8] M. McNeill, S. K. Ali, D. E. Banks, and I. A. Mansi, "Morning report: can an established medical education tradition be validated?" Journal of Graduate Medical Education, vol. 5, no. 3, pp. 374-384, 2013.

[9] C. P. West, J. C. Kolars, C. H. Eggert, C. C. Kennedy, and R. D. Ficalora, "Changing morning report: evaluation of a transition to an interactive mixed-learner format in an internal medicine residency program," Teaching and Learning in Medicine, vol. 18, no. 4, pp. 330-335, 2006.

[10] M. T. James, M. J. Mintz, and K. McLaughlin, "Evaluation of a multifaceted "Resident-as-Teacher" educational intervention to improve morning report," BMC Medical Education, vol. 26, no. 6, article 20, 2006.

[11] A. Khraisat, A. Shanaah, E. AlJaghbeer, D. Berland, and P. B. Cannady Jr., "Morning report emails: a unique model to improve the current format of an internal medicine training tradition," Medical Teacher, vol. 29, no. 4, article 413, 2007.

[12] H. Sanfey, B. Stiles, T. Hedrick, and R. G. Sawyer, "Morning report: combining education with patient handover," Surgeon, vol. 6, no. 2, pp. 94-100, 2008.

[13] M. D. Huffman, S. R. Kaufman, and S. Saint, "A new approach to resident morning report: introducing 'VAVUM,' Internal and Emergency Medicine, vol. 5, no. 1, pp. 81-82, 2010.

[14] K. Layne, A. Nabeebaccus, H. Fok, B. Lams, S. Thomas, and M. Kinirons, "Modernising morning report: innovation in teaching and learning," Clinical Teacher, vol. 7, no. 2, pp. 77-82, 2010.

[15] D. Weaver, "Enhancing resident morning report with 'Daily Learning Packages"' Medical Reference Services Quarterly, vol. 30, no. 4, pp. 402-410, 2011.

[16] G. L. Luciano, B. L. Carter, J. L. Garb, and M. B. Rothberg, "Residents-as-teachers: implementing a toolkit in morning report to redefine resident roles," Teaching and Learning in Medicine, vol. 23, no. 4, pp. 316-323, 2011.

[17] I. I. Bogoch, D. W. Frost, S. Bridge et al., "Morning report blog: a web-based tool to enhance case-based learning," Teaching and Learning in Medicine, vol. 24, no. 3, pp. 238-241, 2012.

[18] S. J. Durning, J. M. Sweet, and L. J. Cation, "Morning report: an analysis of curricular content and comparison to national guidelines," Teaching and Learning in Medicine, vol. 15, no. 1, pp. 40-44, 2003.

[19] R. S. Moharari, H. A. Soleymani, A. Nejati, A. Rezaeefar, P. Khashayar, and A. P. Meysamie, "Evaluation of morning report in an emergency medicine department," Emergency Medicine Journal, vol. 27, no. 1, pp. 32-36, 2010.

[20] B. M. Stiles, T. B. Reece, T. L. Hedrick et al., "General surgery morning report: a competency-based conference that enhances patient care and resident education," Current Surgery, vol. 63, no. 6, pp. 385-390, 2006.

[21] D. E. Kern, P. A. Thomas, and M. T. Hughes, Eds., Curriculum Development for Medical Education: A Six-Step Approach, The Johns Hopkins University Press, Baltimore, Md, USA, 2nd edition, 2009.

[22] IBM Corp, IBM SPSS Statistics for Windows, IBM Corp, Armonk, NY, USA, 2012. 


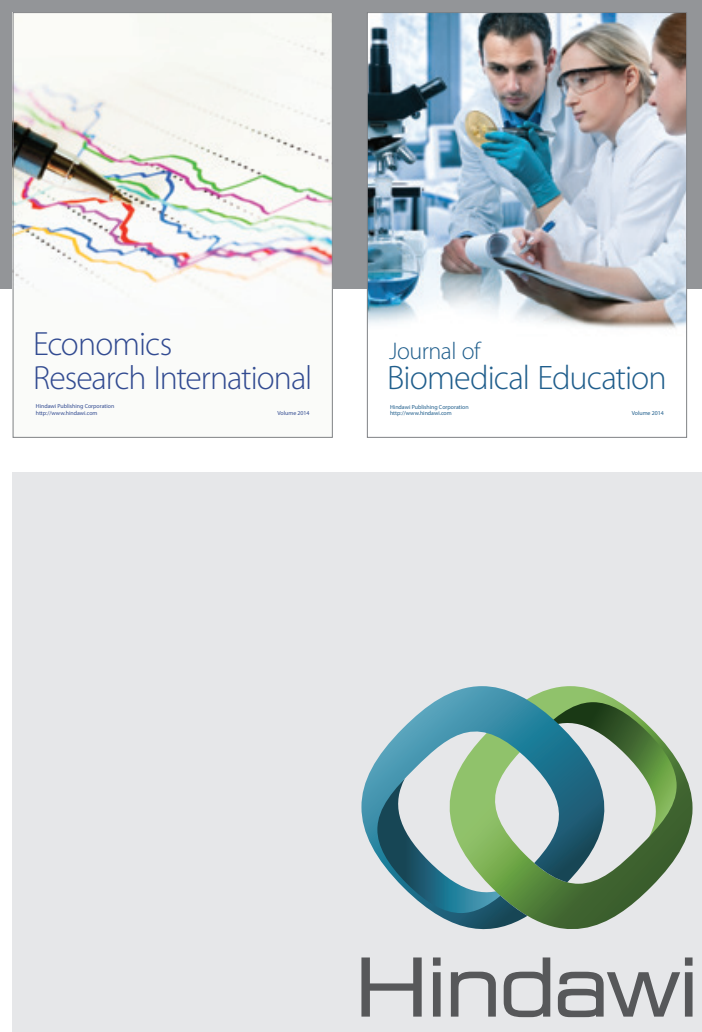

Submit your manuscripts at

http://www.hindawi.com

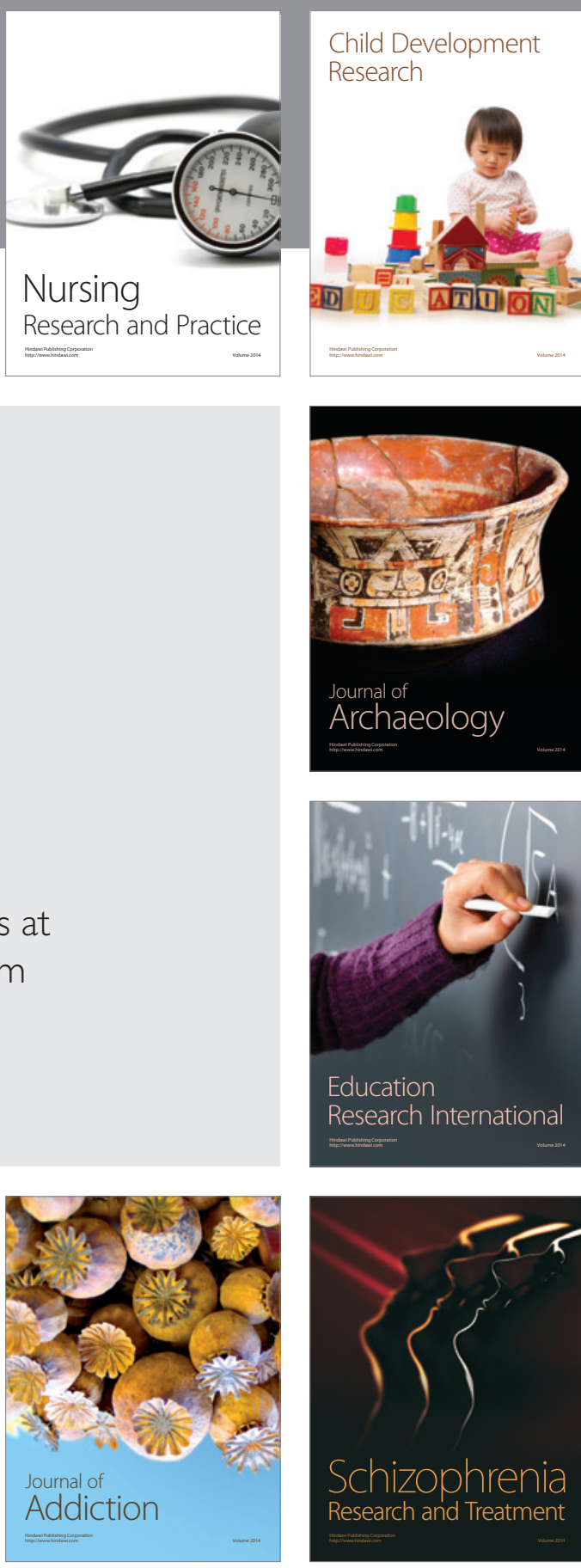

(D)
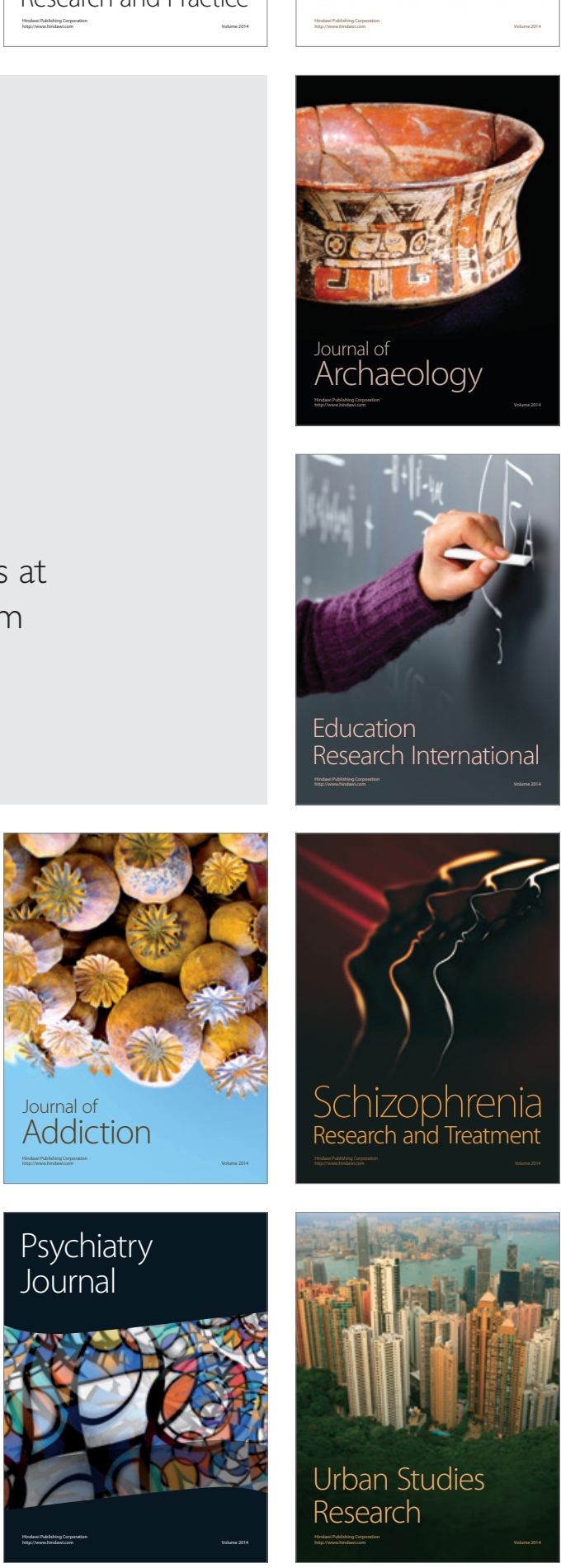